Alcocer Lizcano, J. A. (2013). Competitividad en el sector turístico: una revisión de la literatura. Revista Lebret (5). Bucaramanga, Colombia: Universidad Santo Tomás, pp. 271-291

\title{
Competitividad en el sector turístico: una revisión de la literatura*
}

\section{Competitiveness in the Tourism Sector: a Review of the Literature}

Jaime Armando Alcocer Lizcano

\section{Resumen}

El sector turístico se ha convertido en los últimos tiempos en el centro de la actividad económica de muchas regiones y países del mundo, por lo que ha estado sometido a profundas transformaciones, como resultado de los avances tecnológicos y los cambios en los gustos y preferencias de los consumidores. Las explicaciones sobre los factores que determinan la competitividad de los destinos turísticos se han multiplicado en la literatura académica, como antesala a la necesidad de formular estrategias que apunten al crecimiento y la sostenibilidad de este importante sector de la economía. El objetivo de este artículo es revisar las líneas de debate presentes en esa literatura, con el fin de destacar los factores que pueden servir como referente al momento de plantear estrategias que apunten a generar una mayor ventaja competitiva para el sector.

\section{Palabras clave}

Turismo, Ventaja Comparativa, Ventaja Competitiva, Competitividad.

\section{Código de clasificación JEL: D21}

\begin{abstract}
The tourism sector has recently become the center of the economic activity in many regions and countries of the world, for which it has been subject to profound changes as a result of technological advances and changes the consumers tastes and preferences. The explanations of the factors that determine the competitiveness of tourist destinations have multiplied in the academic literature, as a prelude of the need to develop strategies aimed at the growth and sustainability of this important sector of the economy. The aim of this article is to review the present debate lines in this literature, in order to highlight the factors that can serve as a benchmark when framing strategies aimed at generating a competitive advantage for the sector.
\end{abstract}

\section{Keywords}

Tourism, Comparative Advantage, Competitive Advantage, Competitiveness.

\footnotetext{
* Este artículo es resultado del trabajo de investigación sobre Competitividad del Turismo en el Departamento de Santander, Colombia presentado como requisito parcial de grado en la Maestría en Administración de la Universidad Santo Tomás de Bucaramanga, Colombia

1 Magister en Administración Universidad Santo Tomás, Bucaramanga. Administrador del Sistema SAP FI- GL en Ecopetrol. Correo electrónico: Jaime.alcocer@ecopetrol.com.co
} 


\section{Introducción}

El turismo es uno de los sectores con mayor crecimiento a nivel global en las últimas décadas (Du Toit, Fourie, y Trew, 2010, p.2) y su dinamismo lo ha convertido en el soporte de muchas economías nacionales. Asimismo, las profundas transformaciones que ha traído consigo el proceso de globalización y los cambios notables en las pautas de consumo y las expectativas de los consumidores en todo el mundo lo han sometido, igualmente, a enormes retos y desafíos derivados de una competencia cada vez más profunda - facilitada por el uso intensivo de las tecnologías de la información - y de la imperiosa necesidad de adecuar su crecimiento a los límites de la sostenibilidad ambiental, cultural y social.

En Colombia, el sector ha estado en el centro de atención de la política pública y los planes de desarrollo de los gobiernos recientes, en la medida que se considera que el país tiene un gran potencial relacionado con la diversidad de paisajes y de culturas de sus regiones, como lo ha reconocido el informe del Foro Económico Mundial (Blanke y Chiesa, 2013, p. 19). Sin embargo, de acuerdo con los indicadores usados por esta organización, nuestro país ocupa el puesto 16 entre los 27 países de las Américas en los índices de competitividad del sector, razón por la cual podría afirmarse que está todo por hacerse y que las líneas de política formuladas desde el centro, requieren de programas específicos que a nivel de las regiones, articulen las propuestas de dinamismo turístico en un concepto amplio de desarrollo territorial que privilegie el bienestar de la población y la preservación de las riquezas naturales y culturales (Cohen, 2005).

Aún a pesar de haber sido utilizado con profusión en la literatura académica el concepto de competitividad sigue siendo controvertido, especialmente cuando se trata de definir los factores que permiten la construcción de ventajas competitivas y su conservación en el tiempo, como también a la hora de establecer indicadores que permitan su operacionalización y medición en situaciones concretas. Por tanto, este es un tema abierto y promisorio para la investigación en el campo de las ciencias económicas y administrativas, máxime cuando los modelos de desarrollo de la mayoría de los países se estructuran a partir de la necesidad de alcanzar posiciones exitosas en el complejo escenario internacional de la globalización. Para el caso del turismo, considerado como un sector de potencial desarrollo en la mayoría de los países del mundo, incluida Colombia, el análisis de la competitividad resulta de la mayor importancia dado que, como afirman González y Mendieta $(2009,112)$ esta categoría "aún necesita encontrar un cauce de definición y operativización para su aplicación" en este campo. Para continuar presentando la perspectiva de los autores precitados, es imprescindible acercarse a la "conceptualización y los aportes teóricos sobre el tema en los últimos años, para identificar aquellos que más se adecúan a los fines de entender y guiar el análisis de la competitividad de los destinos turísticos" (p. 112). Es ese, entonces, el objetivo de este ejercicio de reconocimiento de la literatura más reciente sobre el tema que nos ocupa, ejercicio que sirvió de base para 
el desarrollo de una investigación sobre la competitividad de los destinos turísticos en el departamento de Santander (Colombia).

Así, en el presente artículo se hace una revisión de documentos académicos publicados entre 2007 y 2012 en los que se visualiza el debate reciente sobre los factores determinantes de la competitividad en el sector turístico, con el fin de establecer posibles derroteros de investigación sobre el particular, aplicables a las características del sector en países como Colombia. Para contextualizar la presentación de la literatura, el artículo se inicia con una mirada sobre la discusión teórica acerca de las ventajas comparativas y las ventajas competitivas como explicación del comportamiento de las actividades económicas y culmina con algunas observaciones que ratifican la tesis de Esser, Hillebrand, Messner y MeyerStamer (1996) cuando afirman que:

La competitividad no surge espontáneamente al modificarse el contexto macro ni se crea recurriendo exclusivamente al espíritu de empresa a nivel micro. Es más bien el producto de un patrón de interacción compleja y dinámica entre el Estado, las empresas, las instituciones intermediarias y la capacidad organizativa de una sociedad (p. 40).

\section{De las ventajas comparativas a la ventaja competitiva}

\section{La ventaja comparativa como explicación de la competencia}

Se puede afirmar que la llamada teoría clásica del comercio internacional, que intentó explicar, a principios del siglo XIX, los patrones del intercambio entre países, es el primer acercamiento al concepto de competitividad. Dentro de ella, el enfoque de la ventaja comparativa, generalmente asociado con la obra de David Ricardo (1959) pero relacionado también con contribuciones de Smith (2005) y Stuart Mill $(1985)^{2}$, explica la especialización productiva de los países a partir de la presencia de factores naturales como la situación geográfica, el clima o la presencia de materias primas, aunque también se mencionan la maquinaria y la mano de obra. Según Smith (2005), un país debe especializarse en aquel bien que produce más eficientemente, es decir, para el que tiene una ventaja absoluta. Para Ricardo (1959, p. 101) la situación ideal era aquella en la que cada país producía "aquellos artículos que, debido a su clima, su situación y demás ventajas naturales o artificiales, le son propios, o intercambiándolos por los producidos en otros países, como aumentarlos mediante un alza en la tasa de utilidades".

2 Adam Smith publicó La Riqueza de las Naciones en 1756; en 1817, David Ricardo publica Principios de economía política y tributación y en 1848, Stuart Mill publicó Principios de economía política, constituyéndose en las tres obras pioneras de este enfoque. 
Tal como afirma Flores (2009, p. 52) "mientras que Smith cuando se refiere a la ventaja absoluta realiza una comparación entre las ventajas que tiene un país con respecto a otro para producir un determinado producto, Ricardo hace referencia, mediante el concepto de ventaja comparativa, a la diferencia existente entre países a la hora de producir diferentes mercancías". De esa forma, las diferencias en la productividad del trabajo entre países son, según la explicación ricardiana, el núcleo de la especialización productiva y, por tanto, el factor determinante en la estructura del comercio mundial.

En el período comprendido entre las dos guerras mundiales, la teoría de la ventaja comparativa fue complementada y reformulada desde la vertiente neoclásica, mediante el llamado teorema de Heckscher-Ohlin que explica la orientación y composición del comercio internacional por las ventajas comparativas derivadas de la abundancia relativa de los factores de producción de cada país (Alonso, 2011). Así, la localización empresarial y los flujos comerciales se deben a que los factores de producción están distribuidos en proporciones desiguales $\mathrm{y}$, por tanto, tienen costos diferenciados entre países. Un país exportará los bienes que dependen de la utilización intensiva del recurso relativamente abundante e importará aquel que se sustenta en aquellos recursos de los que carece.

El pensamiento neoclásico prevaleció hasta bien entrado el siglo pasado. Sin embargo, las profundas transformaciones derivadas de la revolución tecnológica sustentada en la microelectrónica del reordenamiento geopolítico de las últimas décadas y, especialmente, de la cada vez más profunda interconexión de las economías en lo que se ha denominado el proceso de globalización, terminaron por desvalorizar esta corriente interpretativa la cual, para decirlo en palabras de Porter (1990, p. 170) "ha sido eclipsada en los sectores y países avanzados por la mundialización de la competencia y por la fuerza de la tecnología”.

Ese entorno internacional marcado por cambios permanentes y desafíos crecientes significa un reto para las unidades productivas, pero también para las regiones y los países, que tendrán que superar la visión tradicional para transitar por los nuevos derroteros delimitados por la competencia y los nuevos jugadores internacionales. Así las cosas, se ha gestado una perspectiva renovada acerca de los determinantes de la competitividad que ha permeado el debate académico en áreas muy diversas durante los últimos años.

Sin embargo, lo anterior no significa que sea necesario desechar por completo los principios de la teoría de la ventaja comparativa, ya que está demostrado que cuando se trata de sectores estrechamente relacionados con el territorio - como el turismo algunos de ellos siguen teniendo validez, especialmente si se piensa el valor que tiene en la dinámica turística elementos tales como el clima, la ubicación y los accidentes geográficos, el paisaje, las tradiciones culturales y demás. Como lo plantean Barroso y Flores (2006): 
En este sentido, según Porter (1990) la teoría de la ventaja comparativa va a tener una mayor importancia a la hora de explicar el éxito de determinados países en aquellos sectores o subsectores que utilizan de forma más intensa factores productivos básicos como recursos naturales, mano de obra poco cualificada, importancia de la situación geográfica, entre otros (p.12).

\section{Competitividad y ventaja competitiva}

El concepto de competitividad - de uso creciente en la literatura económica y administrativa - se utiliza para referirse no sólo al desempeño de las empresas sino para hacer relación al comportamiento de sectores productivos, naciones o regiones y constituye el núcleo central de las teorías contemporáneas del comercio internacional y del desarrollo territorial (Guerrero, 1996).

En su versión moderna, las categorías de competitividad y ventaja competitiva tienen su origen en los trabajos del profesor Michael Porter de la Universidad de Harvard. Este autor plantea una línea de investigación que se conoce como la de la competitividad estructural, cuyo núcleo central apunta a relacionar la competitividad de las organizaciones con el entramado de influencias que determinan los factores del entorno económico, las políticas del gobierno y los rasgos internos de la unidad empresarial. Para decirlo en palabras de González y Mendieta (2009, p. 114) "la competitividad nacional se sostiene así en las condiciones macroeconómicas de un país, la sofisticación de las operaciones de las compañías y la calidad del ambiente microeconómico de los negocios en el cual las empresas compiten”.

Según Porter (2005, p. 28) competitividad es "la productividad con la que un país utiliza sus recursos humanos, económicos y naturales". Por una parte, en esta definición se puede reconocer que la productividad, desde el punto de vista de Porter, es uno de los elementos centrales en la obtención de la competitividad. De acuerdo con este autor, "la productividad es el principal determinante, a la larga, del nivel de vida de una Nación porque es la causa radical de la renta nacional per cápita" (Porter, 1991, p. 29) y explica que el estándar de vida de una Nación depende de la capacidad de sus empresas para obtener niveles altos de productividad. A su vez, la obtención de un alto y sostenido nivel de vida constituye el principal objetivo de una nación, de manera que la productividad está directamente relacionada con la competitividad.

Al comparar esta concepción con las teorías convencionales del comercio internacional a las que hicimos referencia en los párrafos precedentes, Sobrino (2002, p. 26) plantea que:

Michael Porter enfatiza menos en la ventaja comparativa como elemento estático que explica el intercambio, y más en la ventaja competitiva que se relaciona con atributos específicos de los países, como son la dotación de factores y tecnología, la producción con rendimientos crecientes a escala, la diferenciación de productos y la homogeneización en los patrones internacionales de consumo. 
De acuerdo con el autor que se está reseñando, la competitividad, en la medida en que se relaciona directamente con la productividad, se puede crear o construir. De ahí la importancia de emprender estudios para determinar el nivel competitivo de sectores o regiones con el fin de que sirvan de base para el diseño de estrategias que conduzcan a un mejor posicionamiento en los complejos contextos actuales. Ahora bien, para llegar a ser competitivas a través de una productividad alta y creciente, las empresas y las economías nacionales a las que están vinculadas dependen de su capacidad para mejorar a través de la innovación tecnológica, capacidad que surge de los retos representados por los competidores y por los consumidores (Wang WenCheng, Lin Chien-Hung y Chu Ying-Chien, 2011).

En el modelo de Porter los determinantes de las ventajas competitivas se representan mediante un diamante de cuatro aristas, a saber: las condiciones de los factores, las condiciones de la demanda, los sectores relacionados y de apoyo, y la estrategia de la empresa, su estructura y la rivalidad con otras unidades empresariales (Porter, 1990). Tales determinantes están estrechamente relacionados e inciden de manera recíproca en sus manifestaciones. De acuerdo con esto, los determinantes de la ventaja competitiva pueden definirse de la siguiente manera:

Las condiciones de los factores se refieren a "la dotación de factores con los que cuenta un país: recursos humanos y físicos, conocimientos, capital e infra-estructura; es decir, los insumos necesarios para competir en una industria" (Hernández-Gracia, 2008 p. 17).

Las condiciones de la demanda se refieren a la demanda interna o externa de bienes o servicios producidos por una empresa y se relacionan con la capacidad de una empresa para captar las necesidades de los consumidores.

Los sectores afines o de apoyo tales como los proveedores de insumos para la producción y de los cuales dependen los bienes o servicios ofrecidos por una empresa, se refieren a la capacidad para generar encadenamientos productivos que mejoren la eficiencia.

La estrategia, estructura y la rivalidad de las empresas: tienen que ver con la manera como se crean y se gestionan las empresas en un contexto dado, y también con la manera como estas compiten en el mercado de bienes y servicios.

Además de los cuatro determinantes, Porter considera dos variables complementarias: el gobierno y los hechos casuales. Este autor hace énfasis en el nivel micro o empresarial como ámbito de consecución de la competitividad y considera que la suma de la competitividad de las unidades empresariales determina, a su vez, la competitividad nacional. Para el caso que nos ocupa, es interesante destacar que, aun cuando Porter no le atribuye un papel determinante al gobierno en la generación de ventajas competitivas ya que su papel se reduce a influir sobre los cuatro determinantes anotados, sin tener capacidad para crearlas por sí mismo, en sus estudios se hace notar la influencia del Estado (Porter, 1991). Esta influencia 
se relaciona con el ambiente empresarial desarrollado a partir de estímulos a la actividad productiva o la formulación de una política pública adecuada para tal fin. En contraposición a Porter, algunos organismos internacionales como la Conferencia de las Naciones Unidas sobre comercio y desarrollo (UNCTAD) considera que "las políticas públicas se constituyen en pilares de la competitividad de un país al ofrecer estabilidad macroeconómica y promover la eficiencia microeconómica" (Sobrino, 2005, p. 131).

La investigación empírica realizada por Porter (2003) lo llevó a comprobar que las empresas líderes en cualquier sector tienden a agruparse en espacios geográficos relativamente pequeños y que su interacción propicia la generación de ventajas competitivas importantes. Estas agrupaciones son conocidas en la literatura como cluster o aglomeraciones y se han convertido en los últimos años en objetivo de política pública en la mayoría de los países del mundo, asumiendo que su consolidación constituye uno de los caminos más acertados para alcanzar el posicionamiento en los mercados internacionales. Al referirse al papel jugado por las aglomeraciones en el diamante de Porter, Vera y Ganga (2007) afirman que:

Los clusters constituyen uno de los vértices del rombo (sectores afines y auxiliares), pero es mejor verlos como una manifestación de las relaciones existentes entre los cuatro vértices. La forma como se manifiestan esas fuentes de competitividad y cómo interactúan entre sí permite explicar la manera en que hacen las empresas para generar, mantener o perder sus ventajas competitivas (p.316).

Según Porter (1999, p. 201) los cluster pueden definirse como "concentraciones geográficas de empresas interconectadas, suministradores especializados, proveedores de servicios, empresas de sectores afines e instituciones conexas que compiten pero también cooperan". Esta categoría resulta de especial importancia a la hora de analizar la competitividad de las zonas o los destinos turísticos, dado que hace referencia a una multiplicidad de instancias o agrupamientos que integran infraestructuras físicas y recursos naturales con estructuras empresariales de transporte, alojamiento, operación turística, agencias de viaje, entre otras.

Tal es el caso del Motor Valley en los alrededores de Módena, norte de Italia. Según Alberti y Giusti (2012) este caso en particular representa .la manera como el patrimonio cultural y el turismo pueden actuar en sinergia para fomentar la competitividad regional. Los autores identificaron un área geográfica bien delimitada en la cual actúa un grupo de factores relacionados con la industria de las carreras automovilísticas:

[...] (a) recursos y actores industriales (plantas industriales, grandes empresas de manufactura, marcas famosas, pequeños artesanos, etc.), (b) patrimonio industrial (museos corporativos, museos industriales, colecciones, etc.), (c) infraestructura deportiva (pistas de carreras, circuitos, kartódromos, etc.), (d) individuos prominentes (pilotos, diseñadores, creadores, expertos de la 
industria, etc.), (e) eventos (ferias, reuniones, eventos, carreras deportivas, etc.) y (f) iniciativas y paquetes turísticos (Alberti y Giusti 2012, p. 262).

De manera especial, estos autores mencionan al patrimonio cultural de ciudades y regiones como atractivo turístico. Para el caso, se refieren al patrimonio cultural no solamente en términos de la alta cultura, sino también en términos del patrimonio popular e industrial y del patrimonio intangible, el cual se constituye a partir de "los conocimientos, las habilidades y prácticas que fundan la identidad y la dignidad de individuos y comunidades, y cuya reproducción implica la transmisión y el enriquecimiento de capacidades tácitas o informales" (Alberti y Giusti, 2012, p. 263). Los aspectos relacionados con el patrimonio cultural y natural cuentan con una importancia que se debe considerar ya que para el caso de Colombia constituyen parte de sus atractivos turísticos. Estudios pertinentes al respecto son los de Niemczyk (2013), Weaver y Lawton (2007), Lu y Stepchenkova (2012), Jing y Fucai (2011) García Frapolli Ayala-Orozco, Bonilla-Moheno, Espadas-Manrique y RamosFernández (2007) y Torres-Sovero, González, Martín-López y Kirkby (2012).

Cabe anotar que otro enfoque relevante sobre competitividad - que no resulta excluyente con la perspectiva de Porter - fue aportado por los investigadores Klaus Esser y Wolfgang Hildebrand quienes plantean que la competitividad es el resultado de la interacción compleja y dinámica de cuatro niveles económicos y sociales en una Nación. Los cuatro estratos que fundamentan la competitividad, según los autores mencionados, son: el nivel micro, referido a las empresas en su dimensión más básica; el nivel meso, que corresponde al Estado y otros actores sociopolíticos, los cuales inciden en la acción de las empresas a través de políticas de apoyo, del estímulo a la formación de empresas y de procesos de aprendizaje a nivel de la sociedad; el nivel macro que se refiere al contexto macroeconómico y social en que se desenvuelven las empresas y, por último, el nivel meta, que alude a la obtención de competitividad a partir de "la integración social, exigiendo no sólo reformas económicas, sino también un proyecto de transformación de la sociedad” (Esser et al., 1996 p. 5).

Por último, interesa destacar que frente al análisis estratégico promovido por Porter, que enfatiza en los determinantes del entorno a la hora de construir ventajas competitivas, y ante la evidencia de comportamientos diferenciados de unidades empresariales ubicadas en el mismo contexto, se ha desarrollado con fuerza en los últimos años la llamada Teoría de los Recursos y Capacidades que resalta las fortalezas al interior de la empresa como fuente segura de competitividad y desarrollo. La estrategia de la empresa y la utilización que haga de sus factores diferenciadores para responder a los retos del entorno - los que resultan imposibles de duplicar en otras unidades empresariales - se constituyen, según este enfoque, en la fuente primaria de competitividad (Barney, 1991; Grant, 2006; Peteraf, 1993). En una época definida como sociedad del conocimiento, esta perspectiva analítica considera las rutinas, el conocimiento y la cultura organizacional como los elementos básicos explicativos del comportamiento empresarial: "factores como la imagen de 
la empresa, el conocimiento tecnológico, el capital humano o la marca son variables con un peso cada vez mayor en la explicación del éxito empresarial" afirman Navas y Ortiz (2002, p. 164) al referirse al peso específico del capital intelectual en las organizaciones.

Existen, entonces, diferentes definiciones del concepto de competitividad, al igual que múltiples postulaciones sobre los factores que la determinan y condicionan. Sin embargo, todas ellas la conciben como la capacidad de un país, región, sector o unidad empresarial para utilizar eficientemente los recursos con los que cuenta y responder a las demandas de la población. Coincidimos con López, Méndez y Dones (2009, p. 126) cuando afirman que un país, región o unidad territorial:

Será competitivo en la medida en que sus actividades productivas, así como sus habitantes y organizaciones públicas, sociales y privadas, sean en conjunto eficaces, eficientes, emprendedores e innovadores; lo que implica que cuenten con los soportes de infraestructuras, equipamientos, capital humano e instituciones necesarios para aprovechar sus ventajas comparativas, constituyéndolas en competitivas.

Es importante anotar, adicionalmente, que las investigaciones recientes apuntan a destacar, de una parte, el carácter multidimensional y complejo de la competitividad $\mathrm{y}$, de otra, la dificultad para medirla, razón por la cual este es un tema en permanente construcción que requiere de esfuerzos investigativos y de la réplica de estudios empíricos comparados.

\section{Competitividad de los destinos turísticos}

Diversos autores conciben los "destinos turísticos" como un conjunto integrado de experiencias que se brindan al consumidor para su satisfacción a través de los recursos, las políticas, las organizaciones y los servicios que ofrece un determinado territorio. Para este trabajo, se acoge la definición de Valls presentada por Mazaro y Varzin (2008b p. 792): "Espacio geográfico determinado, con rasgos propios de clima, raíces, infraestructuras y servicios, y con cierta capacidad administrativa para desarrollar instrumentos comunes de planificación, que adquiere centralidad atrayendo a turistas mediante productos perfectamente estructurados y adaptados a las satisfacciones buscadas, gracias a la puesta en valor y ordenación de los atractivos disponibles; dotado de una marca y que se comercializa teniendo en cuenta su carácter integral". La anterior definición remite a la necesidad de definir de manera precisa los núcleos, zonas o destinos que van a ser objeto del análisis de competitividad, ya que esta precisión resulta fundamental a la hora de establecer conclusiones y derivar propuestas para el desarrollo futuro.

De la misma forma, se asume con Hassan (2000, p. 241) la competitividad de los destinos turísticos como la "capacidad de un destino para crear e integrar productos con valor añadido que permitan sostener los recursos locales y conservar su posición 
de mercado respecto a sus competidores". Hacemos notar aquí que la capacidad para competir de las empresas del sector se relaciona directamente con la posibilidad de integrarse a otras organizaciones y de aprovechar las características del entorno, debido a que la "experiencia turística" es el resultado de la interacción de múltiples servicios.

\section{Las ventajas comparativas como factor explicativo}

En las últimas décadas, de manera paralela al crecimiento de la actividad turística en todo el mundo y de su posicionamiento como uno de los sectores de mayor potencial económico, se han multiplicado los estudios académicos sobre este sector, a partir fundamentalmente, de tres líneas de investigación que están estrechamente relacionadas. De una parte, se ha destacado el papel del sector como generador de oportunidades de empleo, crecimiento económico y, por tanto, como estratégico en la perspectiva de disminución de la pobreza que afecta a una buena parte de la población mundial.

De igual forma, los estudios apuntan a destacar las profundas transformaciones que ha tenido esta actividad al pasar de un turismo de masas depredador y excluyente a otro marcado por el respeto al medio ambiente, el paisaje y las tradiciones culturales, que debe mejorar la calidad de vida de las poblaciones receptoras. De manera transversal - y como una forma de responder a los enormes retos que estas transformaciones han traído consigo - el debate se ha centrado en las alternativas para construir ventajas competitivas reales y sostenibles en el tiempo, con el fin de hacer propuestas que le permitan a las regiones y a las empresas del sector, utilizar las oportunidades que le brindan las nuevas realidades y blindarse ante una competencia que adquiere cada vez más el carácter de global.

Una primera etapa de ese desarrollo se centró en establecer las particularidades de este sector y la posibilidad de utilizar los referentes conceptuales discutidos en el apartado anterior, cuyo origen puede ubicarse claramente en el análisis del sector industrial, para esclarecer la situación de este segmento de la economía que produce bienes intangibles (servicios). Posteriormente, la literatura muestra un interés creciente por los estudios empíricos que se esfuerzan por aplicar las propuestas modélicas y la utilización de indicadores que puedan derivar en el diseño de estrategias competitivas. Las dos perspectivas de análisis serán destacadas en este documento que pretende acercarse a las tendencias recientes con el objeto de que sirvan de base para evaluar el potencial competitivo del turismo en el departamento de Santander ya que, tal como plantean González y Mendieta (2009, p. 112) "la competitividad es un concepto que, aunque ampliamente desarrollado en la literatura académica, aún necesita encontrar un cauce de definición y operatividad para su aplicación al campo del turismo".

Durante mucho tiempo, la perspectiva macroeconómica de las ventajas comparativas prevaleció en el análisis comparado de las fortalezas de cada país para 
la actividad turística. Según esta percepción:

Los países receptores más competitivos -destinos turísticos- serán aquéllos que dispongan de una mejor dotación de los factores utilizados de forma más intensa en esta actividad, tales como: el clima idóneo, la buena accesibilidad, la mano de obra abundante, la riqueza y variedad de recursos naturales y culturales, etc." (Flores, 2009, p. 65).

De esa forma, el éxito de la empresa turística se relacionaba exclusivamente con las características de la zona geográfica en la que se ubicaba y se veía influenciada por el costo de la mano de obra, la tasa de cambio, el tipo de interés y la posibilidad de desarrollar economías de escala, entre otros factores.

Algunos estudios coinciden en señalar que este enfoque argumentativo tiene validez cuando se trata de explicar la aparición y la dinámica de las etapas iniciales de las zonas turísticas, dado que en esas fases, el territorio y sus condiciones socioeconómicas constituyen factores diferenciadores de enorme importancia. Sin embargo, los adelantos tecnológicos y el proceso acelerado de globalización económica le restan progresivamente importancia a estos factores como determinantes para la localización de la actividad turística y a la vez, como elementos explicativos de su éxito o fracaso. Basta con mencionar la enorme facilidad con la que se viaja hoy en día a cualquier parte del mundo, la posibilidad de "construir" artificialmente ambientesréplica de espacios naturales y la relativa homogeneización de las costumbres en términos de pautas de consumo y de recreación.

\section{Entornos complejos y análisis estratégico}

En las últimas décadas del siglo pasado, el cambio de un entorno relativamente estable y predecible a otro marcado por la complejidad y los altos niveles de incertidumbre incidió en el deterioro de las capacidades explicativas del paradigma sustentado en las ventajas comparativas y dio paso al advenimiento del análisis estratégico, del que forma parte el enfoque estructural de la competitividad formulado por Porter. Puede afirmarse, con Flores (2009) que:

Nos encontramos ante un entorno que experimenta una creciente turbulencia y complejidad, por lo que la teoría de la ventaja comparativa, apoyada en la simple ventaja de costes, cada vez es menos válida para explicar el fenómeno de la competencia, pues la gran mayoría de los supuestos de los que parte esta teoría neoclásica... cada vez se ajustan menos a la realidad actual (p. 91).

A diferencia de la mirada macroeconómica que define el entorno a partir de las fronteras nacionales o territoriales, esta perspectiva considera el contexto específico más inmediato de las empresas para destacar el poder de los proveedores o de los clientes, los rasgos de las empresas competidoras o potencialmente competidoras, los productos sustitutos y los rasgos del sector en el que se ubica la unidad productora. Frente a una empresa estática que acepta las tendencias del entorno, el enfoque 
estructural le asigna a la empresa la posibilidad de diseñar estrategias diferenciales para responder a sus retos o variaciones (Porter, 1990).

Aún a pesar de que en los estudios iniciales de Porter (1990) sobre la ventaja competitiva de las naciones no se contempla específicamente el sector turístico, su equipo de trabajo - conocido con el nombre de Monitor- ha enfrentado en varias ocasiones el análisis empírico de núcleos turísticos definidos geográficamente (destinos turísticos o clusters). Esto obedece a la dificultad de considerar de manera homogénea un "sector turístico", debido a la diferenciación que supone la relación con el medio natural y cultural (Rodríguez y Guisado, 2003). Sin embargo, la aplicación genérica de este modelo al estudio de la actividad turística recibió muchas críticas lo que dio lugar al surgimiento de propuestas concretas que utilizando el marco de referencia de la competitividad estructural, han avanzado en el análisis de las características propias de los destinos turísticos. Los enfoques más relevantes se exponen a continuación:

\section{El modelo de Crouch y Ritchie}

Tal como se mencionó anteriormente, el modelo de la ventaja comparativa pone de relieve el "patrimonio turístico" de un destino con el fin de llamar la atención acerca de las posibilidades de explotación especializada de los recursos según una determinada parcela de las actividades turísticas, a saber, de acuerdo con Sánchez Rivero (2006, p. 6), "turismo de naturaleza, turismo activo, turismo de sol y playa, turismo termal, y demás.”)

Sin embargo, dado que esta propuesta explica de manera limitada la forma como en la actualidad se manifiesta la competitividad de las actividades turísticas, ha sido necesario ampliar la visión ricardiana a través de modelos que incorporan de manera especial los aportes de Michael Porter (1990), específicamente el constructo de la "ventaja comparativa". Podría afirmarse que el modelo de la ventaja competitiva tiene como objeto las acciones de planeación y gestión de las actividades turísticas con respecto a la ventaja comparativa de los destinos turísticos (Sánchez-Rivero, 2006) .

En las dos últimas décadas, se han multiplicado los estudios que intentan acercarse a la definición y medición de la competitividad de los destinos turísticos. Sin embargo, para González y Mendieta (2009):

Los autores que con mayor precisión y amplitud han definido la competitividad de estos espacios fueron Geoffrey Crouch y Brent Ritchie (2000), quienes la refieren como «la capacidad de un país para crear valor añadido e incrementar, de esta forma, el bienestar nacional mediante la gestión de ventajas y procesos, atractivos, agresividad y proximidad, integrando las relaciones entre los mismos en un modelo económico y social (p. 122). 
El punto central de la propuesta explicativa de estos autores es la consideración de la competitividad de los destinos turísticos en el largo plazo como el resultado de la combinación y sinergia entre factores derivados tanto de las ventajas comparativas, como de las competitivas (Crouch y Ritchie, 2000)

En su perspectiva, las primeras se relacionan con aquellos factores de los que está dotado el destino turístico y los que han sido creados por el hombre y pueden ser tanto los recursos heredados o naturales como el paisaje, la situación geográfica o las condiciones climáticas, la flora y fauna o el acervo cultural del destino. Así, clasificaron estos factores en cinco categorías a saber: recursos humanos, recursos físicos, recursos de capital, infraestructura y conocimientos pero, más adelante, incluyeron los recursos históricos y culturales como determinantes en la construcción de ventajas comparativas. Insisten en el carácter dinámico de estos factores que cambian en el tiempo, alterando así las ventajas comparativas de los destinos.

Crouch y Ritchie al destacar la importancia de las dotaciones factoriales iniciales relacionadas con los recursos naturales para determinar el "punto de partida" de un destino turístico afirman que, a diferencia de lo que ocurre en otras actividades productivas en las que la mayoría de las ventajas comparativas se agotan, sin poder ser renovadas, este no es el caso para los destinos turísticos, ya que los turistas disfrutan del paisaje o de la cultura, pero no los consumen, en el sentido convencional del término, a condición que se gestionen de manera eficiente.

Sin embargo, este tipo de ventajas tiende a perder importancia en el largo plazo a la hora de explicar el éxito y la competitividad de los destinos por cuanto estrategias sustentadas únicamente en las ventajas comparativas iniciales que dan origen al destino turístico no son sostenibles en el tiempo. Autores como Camisón y Monfort (1998) sostienen que:

Cuando un destino turístico comienza a entrar en la fase de madurez de su ciclo de vida requerirá acciones positivas encaminadas a rejuvenecer el destino, mediante la sustitución de las ventajas comparativas tradicionales por ventajas competitivas que garanticen la permanencia en el mercado y generen niveles más altos de rentabilidad (p.6).

La Organización Mundial de Turismo (1999) destaca, igualmente, la necesidad de superar la visión convencional que relacionaba el éxito del turismo con las ventajas comparativas, para enfrentar la visión estratégica que plantea la necesidad de convertir esas ventajas iniciales en ventajas competitivas en el largo plazo.

En la perspectiva de Crouch y Ritchie que estamos comentando "las ventajas competitivas se derivan de la capacidad para usar estos recursos con eficiencia y añadirle valor a la actividad en el largo plazo y se relacionan con la auditoría y los inventarios, el mantenimiento, crecimiento y desarrollo y eficiencia y eficacia" (Diéguez-Castrillón, Gueimonde-Canto, Sinde-Cantorna y Blanco-Cerradelo, 2011, p. 104). Alonso Ferreras (2009, p. 40) expresa la profunda incidencia de las ventajas 
competitivas condicionadas por la capacidad de los destinos turísticos de gestionar eficientemente sus ventajas comparativas al afirmar:

Así, un destino turístico que implique a sus habitantes con la posibilidad de explotar económicamente sus recursos, que entienda tanto sus fortalezas como sus debilidades, que desarrolle una política de marketing adecuada y la aplique correctamente, probablemente llegará a ser más competitivo que otro destino que no se haya planteado el papel que el turismo juega en su desarrollo económico y social (resaltado en el texto original).

Desde su postulación inicial en el año 1993, Crouch y Ritchie han venido introduciendo mejoras conceptuales a su propuesta, hasta llegar a una formulación acabada en la que hay algunas variantes importantes. De una parte, los autores plantean que la competitividad no puede separarse, en el caso de los destinos turísticos, de la sostenibilidad. Para decirlo en palabras de Alonso Ferreras (2009, p. 54) en la perspectiva de Crouch y Ritchie "para ser competitivo el desarrollo de un destino debe ser sostenible, no sólo económica y ecológicamente, sino social, cultural y políticamente.

Por otra parte, Crouch y Ritchie tienen en cuenta los factores determinantes relacionados con el Macro Entorno Competitivo (Global) el cual:

Está constituido por aquellas fuerzas globales que cambian la composición y la naturaleza de la práctica turística en el destino, como pueden ser, por ejemplo, el creciente interés por el medio ambiente, los cambios demográficos en los mercados de origen, las cada vez más complejas relaciones entre la tecnología y los seres humanos, la expansión de los regímenes democráticos, etc. (Citado en Sánchez-Rivero, 2006, p. 7).

Consideran también estos autores el Micro Entorno Competitivo, que se refiere a las condiciones inmediatas de competencia en que las empresas turísticas deben competir y está conformado por factores tales como los actores locales, las agencias de viajes, las instituciones financieras, la fuerza laboral, la infraestructura hotelera, entre otros. El análisis de los dos tipos de entorno y la respuesta y adaptación a sus condiciones siempre cambiantes, es fundamental para mantener la competitividad de los destinos turísticos.

A continuación, se cita en extenso a Alonso Ferreras (2009) con el fin de destacar la importancia de este modelo para comprender la complejidad de las interrelaciones que se generan entre los factores explicativos de la competitividad de los destinos turísticos, pero también para hacer notar sus limitaciones. Afirma este autor que:

$\mathrm{Su}$ principal aportación es que recoge un amplio conjunto de factores determinantes de la competitividad de un destino estableciendo una categorización inicial de importancia según se trate de factores primarios o secundarios. Sin embargo, los mismos Crouch y Ritchie señalan algunas debilidades de este modelo puramente conceptual como: muchos destinos no disponen de base de datos ni indicadores para evaluar muchos de los 
elementos contemplados; no se pondera el peso de cada uno de los elementos, son necesarias diversas variables e indicadores para evaluar y medir aspectos referidos a atributos naturales y entorno natural de un destino. Además, hasta la fecha, parece que este modelo no ha sido objeto de test empírico adecuado (Ritchie y Crouch, 2003) dada la complejidad de su aplicación empírica en su globalidad (Citado en Alonso Ferreras 2009, p. 57).

\section{Modelo Integrado de Competitividad de destinos turísticos de Dwyer y Kim}

Basados en el modelo de Crouch y Ritchie, Dwyer y Kim (s.f) realizan una reclasificación de las variables que inciden en la competitividad turística y establecen dos grupos de factores que la determinan: los recursos y el entorno o condiciones situacionales. Dentro de los primeros, se consideran los recursos heredados, los recursos creados y los factores y recursos complementarios (Dwyer y Kim, s. f.). Según este modelo, además se deben tener en cuenta los factores de Gestión del destino y las Condiciones de la Demanda, los cuales constituyen en conjunto las llamadas por los autores "condiciones situacionales" (Dwyer y Kim, s. f.). En la siguiente figura, se resumen los elementos de este modelo:

Figura 1. Modelo Integrado de la Competitividad de un Destino Turístico de Dwyer y Kim

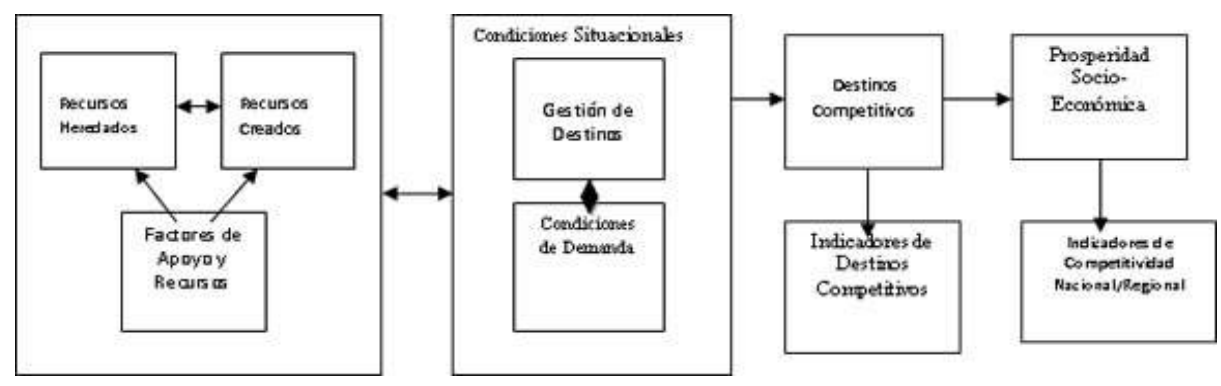

Fuente: Tomado de Dwyer y Kim (s. f., p. 4) (traducción del autor)

Para los autores en mención, los recursos heredados (naturales y culturales), los creados (infraestructura turística que incluye alojamiento, transporte, restauración, entre otros) y los factores o recursos de apoyo (infraestructura general del destino, calidad del servicio, la hospitalidad y demás) son los factores que hacen atractivo un destino y, por tanto, son la base de su competitividad. 
Según estos autores, las condiciones situacionales pueden incrementar o disminuir la competitividad de un destino y se relacionan fundamentalmente con tres elementos: las condiciones para el desarrollo (ubicación geográfica, micro-entorno competitivo y seguridad, entre otros) las condiciones de demanda (percepciones y preferencias, imagen positiva o negativa del destino) y la política o gestión del destino que abarca todas las actividades que tienen como objetivo mejorar los atractivos del destino con el fin de que se adapten a las condiciones situacionales. La competitividad del destino, según el modelo, influye sobre la prosperidad regional/nacional lo cual, a su vez, indica que la competitividad es un logro que apunta hacia el objetivo más amplio orientado al bienestar socioeconómico de los residentes.

En términos generales, puede afirmarse que este es un modelo que apunta a establecer el posicionamiento competitivo del destino, por comparación con otros destinos, en términos de fortalezas y debilidades y recurre a estrategias como la opinión de grupos de expertos o encuestas para establecer las preferencias de los consumidores turísticos o su percepción sobre la calidad de los servicios ofrecidos.

\section{A modo de conclusión}

Es posible afirmar que los profundos desafíos a los que está sometida la actividad turística en todo el mundo, ocasionados por las transformaciones en los gustos y exigencias de los consumidores, por la competencia internacional cada vez más compleja y por las exigencias que se le plantean hoy a los modelos de desarrollo, convierten el estudio sobre los factores determinantes de la competitividad de los destinos turísticos en un aspecto medular que debe ser objeto de reflexión por parte de la comunidad académica y los actores comprometidos directamente en esos procesos.

El análisis de los modelos reseñados en el artículo, que han estado en la base de la gran mayoría de los estudios sobre competitividad turística en las últimas décadas, nos confirma la importancia que tienen las ventajas comparativas para explicar el desarrollo y éxito inicial de los destinos turísticos. Pero también, muestra la necesidad de considerar como determinantes de competitividad, en el marco de un entorno global caracterizado por la creciente complejidad, aquellos factores relacionados con el valor añadido por la acción del hombre en el manejo eficiente de los recursos naturales y culturales heredados. Así, en la situación actual es preciso adoptar la visión estratégica - basada en la categoría de ventajas competitivas- que reconoce los recursos existentes en el territorio como factores importantes para impulsar el éxito del destino, pero asume como determinante su potencial valorización a través de las habilidades de gestión y el uso eficiente de esos recursos.

Importante también destacar que, ante el panorama de deterioro ambiental y cultural provocado por el turismo de masas depredador practicado durante el siglo pasado, los modelos contemporáneos manejan un concepto de competitividad 
vinculado estrechamente a la sostenibilidad social, ambiental y cultural (Crouch y Ritchie, 2000) o al bienestar de la población, en un acercamiento al moderno enfoque del desarrollo local y regional (Dwyer y Kim, s.f.). Lo anterior significa que, a partir de ahora, el éxito de los destinos turísticos deberá medirse también, y prioritariamente, por su capacidad para minimizar los daños ambientales y potenciar los recursos culturales y sociales de los espacios territoriales en los que se ubiquen, lo cual significa que el turismo responsable se convierte en la más válida estrategia de desarrollo del sector.

Igualmente, de este recorrido es posible derivar el énfasis puesto por los analistas en una dupla que podría explicar, en un alto porcentaje, las fortalezas de los destinos turísticos maduros: la gestión empresarial eficiente de los recursos existentes en el territorio y una política pública que incentive la actividad y abra espacios adecuados para que la población local se beneficie de los turistas sin poner en peligro sus referentes culturales. Por tanto, la competitividad se concibe como el resultado de la dinámica integrada de los actores públicos, privados y la ciudadanía, en la que juegan a la par los factores de microeconómicos y los condicionantes del entorno regional y global.

Por último, es necesario reconocer que, la profusión de la literatura sobre el tema que nos ocupa, ha demostrado la complejidad conceptual y metodológica del concepto de competitividad cuando se aplica a un servicio caracterizado por un entramado de múltiples factores y, por tanto, las dificultades de su medición y aplicación en estudios empíricos concretos. De ahí que las investigaciones más recientes se han encaminado a encontrar indicadores adecuados que reflejen esos niveles de complejidad y permitan materializar mediciones de competitividad que enfaticen sobre los aspectos básicos o esenciales sobre los que reside verdaderamente el éxito competitivo de un destino turístico. La discusión sobre esos indicadores se sale de los límites temáticos de este artículo (Alonso Ferreras, 2009).

\section{Referencias}

Alberti, F. G., y Giusti, J. D. (2012). Cultural heritage, tourism and regional competitiveness: The Motor Valley cluster. City, Culture and Society, 3(4), 261273. doi:10.1016/j.ccs.2012.11.003

Alonso, J. A. (2011). Lecciones sobre economía mundial: introducción al desarrollo y a las relaciones económicas internacionales. Madrid: Thompson Civitas.

Alonso, V. H. (2009). Un modelo Integral para evaluar la competitividad de destinos turísticos basado en la identificación de factores críticos de éxito Tesis Doctoral Universidad Las Palmas de Gran Canaria.

Alonso Ferreras. (2010). Factores de éxito y evaluación de la competitividad de destinos turísticos. Estudios y Perspectivas en Turismo 19 (2), 201-220. 
Barney, J. (1991). Firm resources and sustained competitive advantage. Journal of Managemente, 17(1), 99-120.

Barroso-González, M., y Flores-Ruiz, D. (2006). La competitividad internacional de los destinos turísticos: del enfoque macroeconómico al enfoque estratégico. Cuadernos de Turismo, (17), 7-24.

Blanke, J., y Chiesa, T. (2013). The travel y tourism competitiveness report 2013. En Geneva, Switzerland: World Economic Forum. Disponible en: http://mycbs.biz/ data/WEF_TT_Competitiveness_Report_2013.pdf

Camisón, C. y Monfort Mir, V. (1998): Estrategias de reposicionamiento para destinos turísticos maduros: el caso de la Costa Blanca. Estudios Turísticos, (135), 5-28.

Cohen, E. (2005) Principales tendencias en el turismo contemporáneo. Política y Sociedad, 42 (1), 11-24

Crouch, G. I., y Ritchie, J. R. B. (1997). Destination competitiveness and the role of the tourism enterprise. En Proceedings of the Fourth Annual Business Congress. Presentado en Fourth Annual Business Congress, Estambul. Disponible en: http://www.ucalgary.ca/files/haskaynefaculty/95.19.pdf

Crouch, G.I. y Ritchie, J.R.B. (2000). The competitiveness destination: A sustainability perspective, Tourism Management, (21), 1-7.

Diéguez-Castrillón, M. I. D., Gueimonde-Canto, A. G., Sinde-Cantorna, A. I. S., y Blanco-Cerradelo, L. B. (2011). Análisis de los principales modelos explicativos de la competitividad de los destinos turísticos en el marco de la sostenibilidad. CULTUR: Revista de Cultura e Turismo, 5(2), 101-124.

Du Toit, L., Fourie, J., y Trew, D. (2010). The sources of comparative advantage in tourism. Suráfrica: Universidad de Stellenbosch. Disponible en: http://zunia.org/ sites/default/files/media/node-files/wp/187410_wp-01-20101266834241.pdf

Dwyer, L., y Kim, C. (s. f.). Destination Competitiveness: a model and determinants. Current issues in tourism, 6(5). Disponible en: http:/fama2.us.es:8080/ turismo/turismonet1/economia\%20de1\%20turismo/demanda\%20turistica/ DESTINATION\%20COMPETITIVENESS\%20A\%20MODEL\%20AND\%20 DETERMINANTS.PDF

Esser, K., Hillebrand, W., Messner, D., y Meyer-Stamer, J. (1996). Competitividad sistémica: nuevo desafío para las empresas y la política. Revista de la CEPAL, $59,39-52$.

Flores, D. (2009). Competitividad sostenible de los espacios naturales protegidos como destinos turísticos un análisis comparativo de los Parques Naturales 
Sierra de Aracena y Picos de Aroche y Sierras de Cazorla, Segura y las Villas: memoria para optar al grado de doctor. Universidad de Huelva. Disponible en:_http://rabida.uhu.es/dspace/bitstream/handle/10272/184/b15168554. pdf;jsessionid=D55441EA03730C1D6564A777E31D3FB3? sequence $=1 \% 29 \% 20$ consulta\%2005-03-2013

Gandara, J. M. (2004). La calidad y la competitividad de los destinos turísticos urbanos turismo - Visão e Ação, 6 (1) - jan/abril, 69-94

García-Buenahora, M. (2007). Diagnóstico del impacto del proyecto Parque Nacional del Chicamocha sobre el turismo y el ámbito cultural del municipio de Piedecuesta (Tesis inédita de Especialización). Bucaramanga: Universidad Industrial de Santander.

García-Frapolli, E., Ayala-Orozco, B., Bonilla-Moheno, M., Espadas-Manrique, C., y Ramos-Fernández, G. (2007). Biodiversity conservation, traditional agriculture and ecotourism: Land cover/land use change projections for a natural protected area in the northeastern Yucatan Peninsula, Mexico. Landscape and Urban Planning, 83(2-3), 137-153. doi:10.1016/j.landurbplan.2007.03.007

González, R. C., y Mendieta, M. D. (2009). Reflexiones sobre la conceptualización de la competitividad de destinos turísticos. Cuadernos de Turismo, (23), 111-128. Universidad de Murcia

Grant, R. M. (2006). Dirección estratégica: conceptos, técnicas y aplicaciones. Madrid: Thomson Civitas.

Guerrero, D. (1996). La técnica, los costos, la ventaja absoluta y la competitividad. Comercio Exterior, 46(5), 400-407.

Hassan, S. S. (2000). Determinants of market competitiveness in an environmentally sustainable tourism industry. Journal of Travel Research, 38 (3), 239-245. doi:10.1177/004728750003800305

Hernández-Gracia, M. (2008). Los determinantes de la competitividad nacional. Análisis y reflexiones a partir de un marco teórico conceptual. Temas de Ciencia y Tecnología, 12(36). Disponible en: http://www.utm.mx/edi_anteriores/temas036/ ENSAYO2-36.pdf

JJing, Y., y Fucai, H. (2011). Research on management of ecotourism based on economic models. Energy Procedia, 5, 1563-1567. doi:10.1016/j.egypro.2011.03.267

Landínez-Landínez, J. J., y Gómez-Vargas, L. I. (2009). El desarrollo en el Plan de Desarrollo Santander 2008-2011 (Tesis inédita de Especialización). Bucaramanga: Universidad Industrial de Santander.

López, A. M., Méndez, J. J., y Dones, M. (2009). Factores clave de la competitividad regional: inovación e intangibles. Información Comercial Española, ICE: Revista 
de economía, (848), 125-140.

Lu, W., y Stepchenkova, S. (2012). Ecotourism experiences reported online: Classification of satisfaction attributes. Tourism Management, 33(3), 702-712. doi:10.1016/j.tourman.2011.08.003

Mazaro, R. M. (2006). Competitividad de destinos turísticos y sostenibilidad estratégica: Proposición de un modelo de evaluación de factores y condiciones determinantes. Tesis Doctoral presentada al Programa de Doctorado en Investigación y Técnicas de Mercado de la Universidad de Barcelona

Mazaro, R. M., y Varzin, G. (2008a). Competitiveness models for tourist destinations into the sustainability context. Revista de Administración Contemporánea, 12(3), 789-809.

Mazaro, R. M., y Varzin, G. (2008b) Modelos de competitividad para destinos turísticos en el marco de la sostenibilidad RAC, Curitiba, 12 (3), Jul. /Sept.,789809 ,

Mill, J. S. (1985). Principios de economía política: con algunas de sus aplicaciones a la filosofía social (T. Ortiz, Trad.). México, D.F.: Fondo de Cultura Económica.

Niemczyk, A. (2013). Cultural tourists: an attempt to classify them. Tourism Management Perspectives, 5, 24-30. doi:10.1016/j.tmpág.2012.09.006

Ortiz de Urbina Criado, M., y López, J. E. N. (2002). El capital intelectual en la empresa: Análisis de criterios y clasificación multidimensional. Economía industrial, (346), 163-172.

Peteraf, M. A. (1993). The cornerstones of competitive advantage: a resource-based view. Strategic Management Journal, 14(3), 179-191. doi:10.1002/smj.4250140303

Porter, M. E. (1990). The competitive advantage of nations. Harvard Business Review. Disponible en: http://kkozak.wz.cz/Porter.pdf

Porter, M. E. (1991). La ventaja competitiva de las naciones. Buenos Aires: Vergara.

Porter, M. E. (2005). Qué es la competitividad. Apuntes de Globalización y Estrategia, 1(1). Disponible en: http://www.iese.edu/es/ad/AnselmoRubiralta/ Apuntes/Competitividad_es.html

Ricardo, D. (1959). Principios de economía política y tributación. México: Fondo de Cultura Económica.

Rodríguez, M. del M., y Guisado, M. (2003). Competitividad y análisis estratégico del sector turístico de Galicia: consideraciones para la mejora competitiva Revista galega de economía: Publicación Interdisciplinar da Facultade de Ciencias Económicas e Empresariais, 12(1), 145-166.

Sánchez-Rivero, M. (2006). Elaboración de un ranking de competitividad de los 
destinos turisticos españoles: Un análisis provincial mediante modelos de estructura latente. Revista de Análisis Turístico, (1), 4-22.

Smith, A. (2005). La riqueza de las naciones (C. Rodríguez Braun, Trad.). Madrid: Alianza Editorial.

Sobrino, J. (2005). Competitividad territorial: ámbitos e indicadores de análisis. Economía, sociedad y territorio, 99, 123-183.

Sobrino, L. J. (2002). Competitividad y ventajas competitivas: revisión teórica y ejercicio de aplicación a 30 ciudades de México. Estudios Demográficos y Urbanos, 311-361.

Torres-Sovero, C., González, J. A., Martín-López, B., y Kirkby, C. A. (2012). Socialecological factors influencing tourist satisfaction in three ecotourism lodges in the southeastern Peruvian Amazon. Tourism Management, 33(3), 545-552. doi:10.1016/j.tourman.2011.06.008

Valdés, L. (2001): Turismo, desarrollo y sostenibilidad; en Buendía, J. y Colino, J., Eds.: Turismo y medio ambiente 49-72

Valls Josep-Francesc. (2007). Marco estratégico para impulsar el desarrollo bajo criterios sostenibles Estudios Turísticos, (172-173), 175-180

Vera, J. R., y Ganga, F. A. (2007). Los clusters industriales: precisión conceptual y desarrollo teórico. Cuadernos de Administración, 20(33), 303-322.

Wang Wen-Cheng, Lin Chien-Hung, y Chu Ying-Chien. (2011). Types of competitive advantage and analysis. International Journal of Business y Management, 6(5), 100-104.

Weaver, D. B., y Lawton, L. J. (2007). Twenty years on: The state of contemporary ecotourism research. Tourism Management, 28(5), 1168-1179. doi:10.1016/j. tourman.2007.03.004

World Economic Forum. (2008). The travel and tourism competitiveness. Report 2008. Ginebra. 